\title{
Communication
}

\section{Implementing a New Technology in Diagnostic Services for Tuberculosis in Nigeria}

\author{
Amos Oluwasayo Akinremi \\ Department of Planning, Research and Statistics, Ogun State Health Insurance Agency, Abeokuta, Nigeria \\ Email address: \\ amosolusayo@yahoo.co.uk
}

\section{To cite this article:}

Amos Oluwasayo Akinremi. Implementing a New Technology in Diagnostic Services for Tuberculosis in Nigeria. Central African Journal of Public Health. Vol. 6, No. 6, 2020, pp. 358-364. doi: 10.11648/j.cajph.20200606.17

Received: December 3, 2020; Accepted: December 10, 2020; Published: December 25, 2020

\begin{abstract}
This article analyses the experience of the Federal Ministry of Health (FMOH), Nigeria in its partnership arrangement with some international organisations to introduce a new technology to its diagnostic services for tuberculosis (TB) by adopting the use of GeneXpert test in line with the recommendation of World Health Organisation (WHO). It was a major health service reform targeted at achieving the third sustainable development goal. Therefore, the objective of this article was to review the literature on management issues and problems of change surrounding the implementation of GeneXpert as a new technology in the diagnostic services for TB in Nigeria, and provide learning opportunities for health service administrators in resource-limited settings that might be considering such arrangement. A literature survey of articles published between 2008 and 2018 was conducted; using Google Scholar, ScienceDirect and Scopus; to identify and review articles that analyse the change management process in the introduction of GeneXpert test for TB diagnosis in Nigeria. Consequently, a total of 10 articles were critically analysed. The review showed a paucity of articles that examined how change process in the introduction of GeneXpert test for TB diagnosis was managed in Nigeria. The literature survey identified several challenges in the change process such as human resource and capacity building especially because the use of GeneXpert requires a certain level of computer literacy. In conclusion, this review highlighted the fact that the reform might be sustainable because of the adoption of decentralised service system in the implementation process.
\end{abstract}

Keywords: Change Management, Health Service Reform, Public Private Partnership, SDG 3, Tuberculosis, GeneXpert, Decentralised Service System

\section{Introduction}

This article analyses the experience of the Federal Ministry of Health (FMOH), Nigeria in its partnership arrangement with some international organisations to introduce a new technology to its diagnostic services for tuberculosis (TB) by adopting the use of GeneXpert test in line with the recommendation of World Health Organisation (WHO). The Genexpert test is a molecular test for TB that has the potential to revolutionize the diagnosis and care of people with TB [1]. The technological advancements in the operation of GeneXpert machines provide clients the opportunity to have their test results in less than 2 hours [2]. Meanwhile, TB is a deadly disease with significant burden on economy and human development in Nigeria [3, 4]. The essay will raise some of the issues surrounding the implementation of this reform, for example; public private partnerships (PPPs) with relevant stakeholders in the health sector for technical and financial support, procurement and maintenance of the GeneXpert machine, its distribution to health facilities at the community level to ensure decentralised service, training of health personnel to build their capacity on the use of the new technology, data collection and reporting system for performance management, and monitoring and evaluation (M\&E).

The existing Nigeria health service structures in the fight against tuberculosis have been in place for many decades. The effort to improve techniques for diagnosing TB has been very little in the past. The common diagnostic method for TB in Nigeria, which is "smear microscopy", is 125 years old and routinely misses half of all TB cases [5]. Hence, TB tests are 
outdated and inadequate in the country. The primary challenge is that only $16 \%$ of tuberculosis cases are being detected in the country [4, 6]. Therefore, the nation's Federal Ministry of Health identified an urgent need to further build the system to ensure that the structures are able to deal with this challenge; in the spirit of global sustainability and wellbeing. It therefore decided in 2012 [7] to go for a policy change in the diagnostic services for $\mathrm{TB}$ after several consultation and engagement with relevant stakeholders. The essay aims at developing a detailed understanding of management issues and problems of change surrounding the implementation of GeneXpert as a new technology in the diagnostic services for TB, and provides learning opportunities for health service administrators in resource-limited settings that might be considering such arrangement.

Analytical description of the organisation:

The delivery of TB services has undergone enormous transformation in recent decades, with new organisational forms emerging as an organ of FMOH. In I988, FMOH created the National Tuberculosis and Leprosy Control Programme (NTBLCP) within the Department of Public Health, with the aim of eliminating TB and leprosy. The organisational structure of NTBLCP is such that it has a National Coordinator as the head, supported at the federal, state and local government levels by a team of medical officers, laboratory scientists and other support staff [8]. The fight against tuberculosis has always been a collaborative effort as it involves joint efforts from governments, communities, civil society organisations and many development partners and international organisations. Meanwhile, it has been indicated that efficiency, flexibility and value for money might be guaranteed with strong collaboration among actors from both public and private sectors [9]. This practice or arrangement of 'hybrid' governance like PPPs is different from contracting-out or outsourcing because PPP has to do with risk sharing and co-production [10] while contracting-out or outsourcing is a temporary business relationship in which the government retains control and ownership. The problem with this type of arrangement as reported is the delay experienced during the negotiation stages which often results into huge advisory cost overruns [11].

FMOH used public private partnership concept as a procurement strategy in the fight against TB because in this type of arrangement private organisations initially have to finance the programme, and this offers a form of relief to national government. In early 2013, for example, a grant from the Centre for Disease Control (CDC) was used to procure some GeneXpert machines [12]. The cost of GeneXpert is challenging in resource-limited areas, and PPPs assist and reduce pressure on government budgets, even though this may be temporary and unsustainable.

Goals/impetus for the reform:

This is a major health service reform targeted at achieving sustainable development goal (SDG) 3. The third SDG is to ensure healthy lives and promote well-being for all at all ages; with a specific call to end the epidemics of HIV, TB and malaria by 2030 [13]. The target proposed is a $90 \%$ reduction in tuberculosis deaths and an $80 \%$ reduction in new cases by 2030. To achieve these milestones, novel tools and approaches for finding tuberculosis cases is essential, as well as expanding access to tuberculosis services, through health systems strengthening, particularly at the primary care level. In 2010, WHO recommended the use of GeneXpert test for the diagnosis of tuberculosis globally because of the failure of "smear microscopy" to detect cases of tuberculosis in some situations such as human immunodeficiency virus (HIV) and drug resistance cases, and Nigeria adopted the use in 2012. This may therefore be analysed as a case of policy transfer which leads to a policy change or policy reform.

\section{Methods}

A literature survey of articles published between 2008 and 2018 was conducted; using Google Scholar, ScienceDirect and Scopus; to identify and review articles that analyse the change management process in the introduction of GeneXpert test as a new technology in tuberculosis (TB) diagnostic services in Nigeria. Literature review was considered for this study a proper research design seeing that there was dearth of studies which critically analyse how the change process of introducing GeneXpert in Nigeria was managed. The process of the review followed the sequential pathway of finding the research question; locating relevant research articles; picking the research articles for review; summarising and documenting; and organising, analysing, synthesising and reporting findings. Key themes such as stakeholders engagement, change environment, implementation structure, challenges of change, tasks and functions, and monitoring and evaluation were adapted and put together to develop an understanding of change management process in relation to the introduction of GeneXpert test in Nigeria.

\section{Results and Discussion}

A total of 10 articles were critically analysed. The review showed a paucity of articles that examined how change process in the introduction of GeneXpert test for TB diagnosis was managed in Nigeria, and the key themes identified were discussed below:

\section{Stakeholders consultation and engagement:}

The NTBLCP, led by its national coordinator, went into a strong consultation and engagement to gain support and the ensure success of this change process as many stakeholders were involved in the roll out of this new method of diagnosis. The professional bodies of doctors, nurses, laboratory workers, and other health workers were fully consulted from the planning stage to avoid resistance to the proposed change because they form the staff strength in the hospitals where the new technology will be deployed. NTBLCP convened stakeholders meeting that had in attendance people from diverse backgrounds, cultures, beliefs, values, norms, institutions, and professional affiliations; including the medial professionals who helped to create awareness and community 
sensitization. The Honourable Minister for Health at one of the stakeholders meeting emphasized the need for the introduction of GeneXpert in order to create readiness for change among stakeholders. He analysed the impact of TB on economy and human development to justify the need for the new technology. Meanwhile, it has been noted that readiness for change is reflected in the beliefs, intentions and attitudes of an organisation's members regarding the extent to which changes are needed and the capacity of such organisation to effectively implement those changes [14]. The media helped to disseminate a lot of information as regards the operation of GeneXpert. This is worth noting because though process changes might appear simple, it is actually complex as many policies may be affected [15]. Hence, it usually calls for a lot of consultation for example with labour unions and the public in general, which is a very long process.

The change environment:

Some external pressure in terms of political, economic, technology and social factors influenced the change for GeneXpert. The limitation of "smear microscopy" as a TB diagnostic service met with international politics which can be demonstrated by WHO's call for the introduction of a better alternative method. A major challenge to the control of tuberculosis is a diagnostic process that requires multiple visits with consequent expenses from clinic fees and transport. Most patients attend clinics with companions, thereby increasing transport fee. High expenditure has been found to be associated with attending clinics with company, residing in rural areas far from diagnostic centres and illiteracy [16]. The costs usually incur by patients are very substantial. It is therefore important that clients get value for money. This is achievable with the introduction of GeneXpert as the new diagnostic service for TB. Meanwhile, it has been argued that immediately an agenda receives attention, it will spread very quickly and become impossible to stop or prevent [17]. On the other hand, punctuations occur and are attributed to external incidents that disturb the political regime, especially those that are sufficiently huge to disrupt the equilibrium. In this case, the political system responded to the challenge of increasing burden of TB on the country's socio-economic development, and the external influence or pressure that came in form of recommendation from WHO. It was indicated that when agenda-setting and policy formulation are international in origin, institutions play an essential role as epistemic community in the political stream of public policies [18]. For example, WHO, United States Agency for International Development Agency (USAID), Department for International Development (DFID), Centre for Disease Control (CDC) and other international organisations have always been supporting the "STOP TB Strategy". The aim of the network is to comprehensively strengthen the detection of TB, and eventually eliminate the disease.

Structure of the implementation arrangement:

The FMOH adopted a decentralised form of governance in its implementation arrangement to introduce GeneXpert test as a new technology in the diagnostic services of TB because it is not restricted to central or reference laboratories, which was done in the case of some conventional diagnostic tests. The
GeneXpert machines were distributed to community health facilities to bring the service closer to the people and expand access to the service. The facilities were given as much information and resources as possible to empower them and give them a sort of autonomy to manage the system. This type of arrangement will increase local accountability because of its closeness to people which will give everyone the chance to contribute to the process of implementation. It will consequently create a system that is effective, efficient and capable of responding to the needs of the populace. It has been noted that health services might be better when delivered by healthcare organisations that have greater autonomy than by organisations under closer central and political control [19]. The argument against decentralisation is the financial implication as it was suggested that governance could be undertaken from the centre more cheaply [20]. However, it was argued that decentralised GeneXpert test implementation is feasible and could lead to an improvement in tuberculosis care and control [21]. A report from India indicated that GeneXpert can be deployed at the decentralised level for all TB suspects in diverse settings of the country [22]. The experience in Nigeria confirms a better service delivery with decentralised system. The closer the diagnostic service for TB is to people the better the access because of reduced waiting and travelling time. It is economically safe for the end users to have the service close to them to overcome the barrier of distance and transport fare. Nickols, however, argued that successful change is based on building a new organization and gradually transferring people from the old one to the new one in an incremental manner rather than a radical approach; to give people time to adapt to the new circumstances [23]. This is especially good for the health workers who will be using the new technology, because they are going to be learning new things, and they need time to get full understanding of the new system.

Meanwhile, NTBLCP implemented the introduction of GeneXpert test as TB diagnostic service in phases (as shown in Figure 1); to effectively manage the change process. The implementation pathway is divided into nine phases with 22 steps. The first is "introduction" phase, and it consists of steps $1-5$ as labelled below. The expected outcome is the establishment of planning and coordination body; by the name "TWG" (Technical Working Group). The second is "strategic planning" phase, and it consists of steps 6-10. The expected outcome is the development of draft national GeneXpert strategic plan. The third is "site assessment" phase, and it consists of step 11. The expected outcome is the collection of all required information for finalization of strategic plan and the annual activity plan. The fourth is "finalization of strategic plan" phase, and it consists of steps 12-15. The expected outcome is the finalization of national GeneXpert strategic plan. The fifth is "preparation" phase, and it consists of step 16. The expected outcome is the completion of laboratory renovations and development of documents and laboratory support systems such as maintenance, supervision, etc. The sixth is "training and installation" phase, and it consists of steps 17-18. The expected outcome is the commencement of routine use of GeneXpert. The seventh is "routine monitoring 
and supervision" phase, and it consists of step 19. The expected outcome is the assurance of quality of GeneXpert use. The eighth is "evaluation" phase, and it consists of steps
20-21. The expected outcome is the informed national policy and practice through collected evidence and experience. The last is the "scale up" phase.

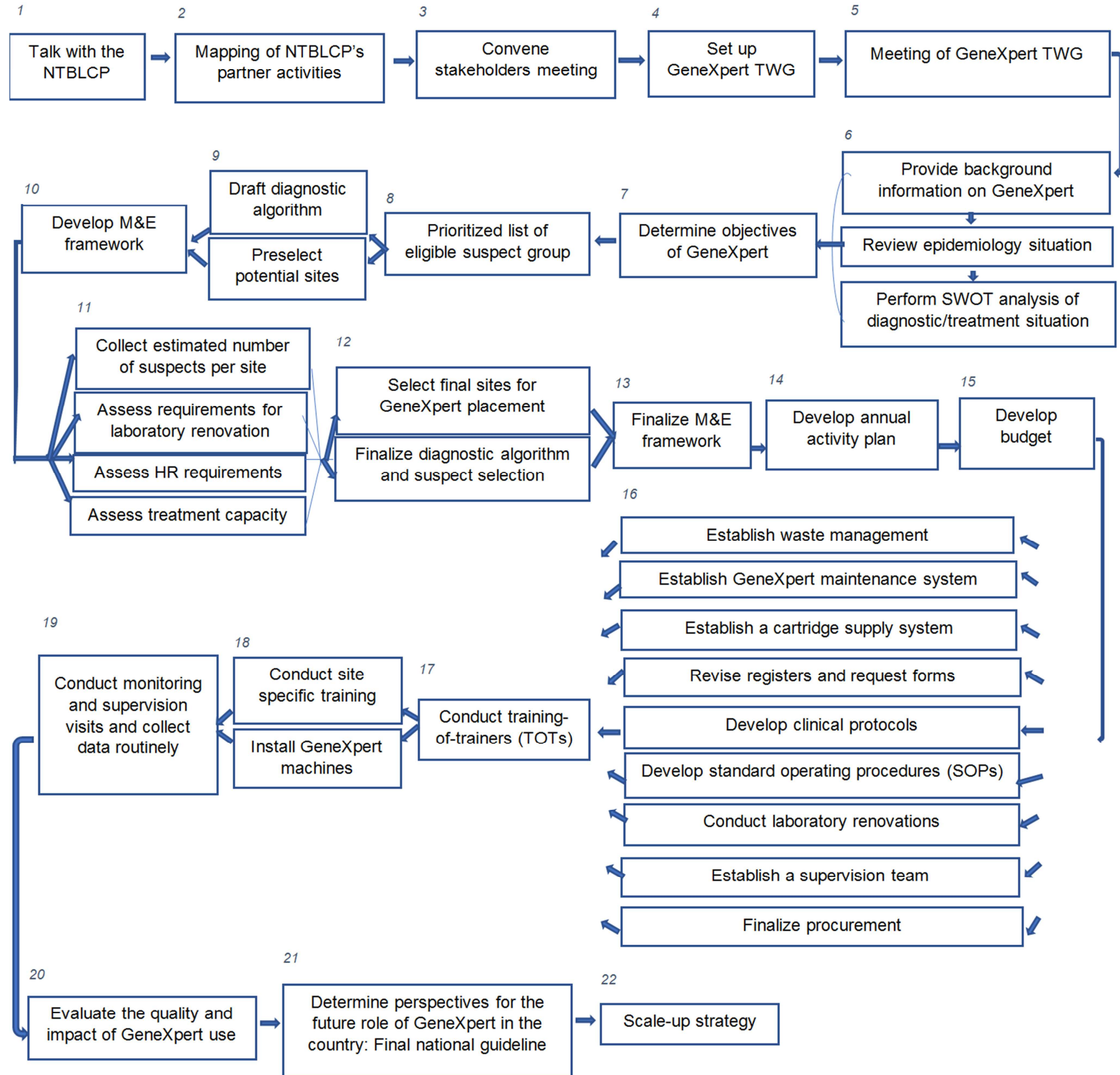

NB: "SWOT" stands for Strengths, Weaknesses, Opportunities and Threats.

Figure 1. GeneXpert Implementation Pathway (adapted from USAID and TB CARE I, cited in [24-26].

\section{Challenges and problems of change:}

The NTBLCP faced some operational, technical and logistic issues in its roll-out of GeneXpert because it was a new technology. There are several anticipated challenges such as human resource and capacity building especially because the use of GeneXpert requires a certain level of computer literacy. It was documented that the GeneXpert system consists of an instrument, personal computer, barcode scanner, and preloaded software, and uses single-use disposable cartridges containing lyophilized reagents, buffers and washes [2]. Therefore, the computer literacy of local staff required extra training [24], and there was need for dedicated personnel who could be trained, to perform testing and keep the machine in good order. Meanwhile, it has been indicated that when employees are confronted with new organisational realities, a sentimental yearning or wistful desire for how things were before the change could be an extremely significant cause of resistance on their part [15]. Hence, decision makers and employers have to employ a number of strategies to create support for change to ensure compliance and avoid resistance.

Tasks and functions:

Strong coordination is required in a PPP arrangement to 
achieve the target goal. FMOH through NTBLCP is responsible for the coordination of the programme at the national level. Overall global coordination of the development partners cannot be overemphasized because most of the partners are international organisations. WHO is therefore responsible for setting the guidelines at the global level. Creswell et al. noted that many international agencies and donors have already expressed interest in investing resources for the roll-out of GeneXpert [2]. Coordination of these activities is essential to optimise the use of available resources, streamline activities, and ensure sound technical advice and approaches at country level. The national GeneXpert TWG are responsible for planning and implementation activities shown in Figure 1. Ceipheid HBDC is the company responsible for the production of GeneXpert device, and it supplies the machine at a subsidized price because of the partnership arrangement [27]. KNCV Tuberculosis Foundation is responsible for the procurement, installation and maintenance of the device. It also does training for health workers on how to operate the device. Global Funds releases grants to support the programme. Civil Society organisations are involved in community sensitization. Health facilities are the service providers.

Monitoring and evaluation:

Monitoring and evaluation can be seen as a communication channel because it gives feedback to programme managers through the data collected. The source of the data is from recording and reporting. Some of the data being collected routinely are number of days in a month that the device could not be operated, and the reasons it could not be operated. Number of tests in a month is monitored as well. Data are also collected on logistic issues such as catridge supply, power stability, etc. These data will allow for the generation of simple indicators to quantify the impact of the new test on laboratory work and diagnosis of TB. However, there is a problem with recording and reporting because monitoring and evaluation system in Nigeria is paper-based. This complicates data collection because the GeneXpert register is sometimes not in place on the field or not properly filled. Nigeria should ideally move completely to an electronic recording and reporting system to generate data that is relevant to the national programme; for the purposes of monitoring and evaluation and direct patient management [24].

\section{Conclusion}

The fundamental problem in low-resource settings like Nigeria, as depicted in the "Piot" model of case finding and treatment, is that individuals seeking care are lost before treatment even begins as they are not properly and promptly diagnosed [28]. It was noted that even with the increasing evidence that a wide range of new diagnostic technologies that exist in the field of TB diagnostics can be used successfully in the most challenging settings; most of the patients that should use these technical evolutions do not yet have access to them [29]. This implies that just the roll-out of an improved TB diagnostic tool like GeneXpert might not be enough to guarantee better outcomes for patients because the processes of implementation within existing health care systems can critically affect the impact. However, there is hope that this reform will be sustainable in the country because of its adoption of decentralised service system by its distribution of GeneXpert machines to health facilities at the community level and giving them autonomy in its management. This is expected to create a system that is effective, efficient and capable of responding to the needs of the populace. Though the use of GeneXpert has gained the support of international partners with discounts negotiated for the test, the concessionary cost is only applicable to non-governmental organisations (NGOs) and the public sector [1]. This implies that it might be difficult accessing TB services at private facilities where a number of people in the country receive health services; thereby limiting access to early and accurate diagnosis, and potentially increasing "morbidity associated diagnostic delay, dropout and mistreatment" [21]. In addition, the fate of GeneXpert funding in Nigeria is uncertain as government of Nigeria is yet to commit to funding the machine in spite of assertions that GeneXpert's ability to offer early diagnosis indicated that its adoption would cost less than current diagnostic devices. There is therefore no doubt that this reform is an ambitious and a demanding one, and its successful implementation requires the engagement and commitment of every relevant stakeholders that are involved in the health system.

\section{Recommendation}

There is need for regular collection and analysis of performance data from TB centres at all levels of care; primary, secondary and tertiary health facilities in the country; to generate evidence on the feasibility and impact of introducing GeneXpert service delivery with decentralised system. On the other hand, government could leverage more on public private partnership to fund procurement and maintenance of the GeneXpert machine as it seems from a pilot analysis that GeneXpert can likely, by as much as $75 \%$, increase TB detection [12]. Meanwhile, health-system barriers to GeneXpert use in the country must be pre-empted and resolved. For example, with procurement of additional machines, there will be need to train additional local staff to perform GeneXpert troubleshooting and maintenance [24]. This is because even the most improved and promising diagnostic technologies will have just minimal impact if they cannot be reached by those who need them. In other words, the actual impact of any diagnostic technologies or interventions depends on the system in which they are going to be deployed. Nigerian health systems therefore must be improved upon to encourage potential clients not to delay care-seeking and to give swift access to proper treatment immediately a diagnosis is received. Furthermore, it is an exigency to have renewed and concerted efforts to maximize the possible responsibilities of governments, development partners, product developers, industries, and NGOs [30] if tuberculosis must be eliminated in Nigeria. Meanwhile, the National TB Programs must provide strong leadership and coordination for the implementation process [24]. In other words, to achieve the full potentiality of promising diagnostic technologies like GeneXpert, various 
groups of stakeholders must give their support to large-scale innovation and delivery while civil society organisations, activists and advocates ought to ensure everyone is held accountable and make sure that publics move the call for improved systems [5]. Lastly, it is essential to have operational and implementation researchers promptly detect and address a wide range of issues that are vital in improving TB services while decision makers are encouraged to convert scientific evidence into policies and guidelines that address the full gamut of issues identified and recommendations from the researchers.

\section{References}

[1] Kanabus, Annabel (2017) "Genexpert Test - TB Diagnosis and Resistance Testing" TBFACTS Information about Tuberculosis, accessed at https://www.tbfacts.org/xpert-tb-test/, 27 December 2017.

[2] Creswell, Jacob; Falzon, Dennis; Getahun, Haileyesus; Gilpin, Chris; Glaziou, Philippe; Grzemska, Malgorzata; de Dieu Iragena, Jean; Lienhardt, Christian; Lonnroth, Knut; Mirzayev, Fuad; Ottmani, Salah; Pantoja, Andrea; Sculier, Delphine; Suvanand, Sahu; Uplekar, Mukund; van Gemert, Wayne; Wares, Fraser and Weyer, Karin (2011) "Rapid Implementation of the Xpert MTB/RIF diagnostic test: Technical and Operational 'How-to' Practical considerations”, World Health Organisation, accessed at http://apps.who.int/iris/bitstream/10665/44593/1/9789241501 569_eng.pdf, 28 December 2017.

[3] Abdurrahman, Saddiq; Mbanaso, Omezikam; Lawson, Lovett; Oladimeji, Olanrewaju; Blakiston, Matthew; Obasanya, Joshua; Dacombe, Russell; Adams, Emily; Emenyonu, Nnamdi; Sahu, Suvanand; Creswell, Jacob; and Cuevas, Luis (2015) "Testing Pooled Sputum with Xpert MTB/RIF for Diagnosis of Pulmonary Tuberculosis To Increase Affordability in Low-Income Countries", Journal of Clinical Microbiology; 53 (8): 2502-2508.

[4] Adejumo, Olusola; Daniel, Olusoji; Abdur-Razzaq, Husseine; Shogbamimu, Yeside; Femi-Adebayo, Toriola; Adepoju, Victor; Adebayo, Bisola and Sodipo, Oluwajimi (2017) “Trend of tuberculosis case notification and treatment outcome in Lagos State, Nigeria: a 5-year retrospective study", Trans $R$ Soc Trop Med Hyg; 00: 1-8, doi: 10.1093/trstmh/trx060.

[5] Small, Peter and Pai, Madhukar (2010) "Tuberculosis Diagnosis - Time for a Game Change" New England Journal of Medicine, 363 (11): 1070-1071.

[6] Vassall, Anna and Mustapha, Gidado (2015) "Post 2015 development agenda; Nigeria perspectives: tuberculosis", Copenhagen Consensus Center, 15 May, accessed at http://www.copenhagenconsensus.com/sites/default/files/niger ia_tb_resource_packet_0.pdf, 5 January 2018.

[7] Akanbi, Maxwell; Achenbach, Chad; Taiwo, Babafemi; Idoko, John; Ani, Agatha; Isa, Yetunde; Agbaji, Oche; Ukoli, Christiana; Akande, Patrick; Maiga, Mamoudou and Murphy, Robert (2017) "Evaluation of gene xpert for routine diagnosis of HIV-associated tuberculosis in Nigeria: A prospective cohort study", BMC Pulmonary Medicine; 17: 87-97.

[8] FMOH (2010) "National Tuberculosis and Leprosy Control Programme (NTBLCP)”, Workers' Manual Revised $5^{\text {th }}$ Edition, Federal Ministry of Health Nigeria.
[9] Willems, Tom and Van Dooren, Wouter (2011) "Lost in diffusion? How collaborative arrangements lead to an accountability paradox", International Review of Administrative Sciences; 77 (3) 505-530.

[10] Shaoul, Jean; Stafford, Anne and Stapleton, Pamela (2012) "Accountability and corporate governance of public private partnerships", Critical Perspectives on Accounting; 23: 213- 229.

[11] Ahadzi, Marcus And Bowles, Graeme (2004) "Public-private partnerships and contract negotiations: an empirical study", Construction Management and Economics; 22: 967-978.

[12] Poole, Brian; Akanbi, Maxwell and Achenbach, Chad (2015) "GeneXpert Implementation for diagnosing TB in HIV patients in Jos, Nigeria", Global Health, 22 September, accessed at http://globalhealth.northwestern.edu/docs/GeneXpertPoster.pd f, 26 December 2017.

[13] Fowkes, Freya; Draper, Bridget; Hellard, Margaret and Stoové, Mark (2016) "Achieving development goals for HIV, tuberculosis and malaria in sub-Saharan Africa through integrated antenatal care: barriers and challenges", $B M C$ Medicine; 14 (1): 202-211.

[14] Armenakis, Achilles; Harris, Stanley; and Mossholder, Kevin (1993) "Creating Readiness for Organizational Change", Human Relations, 46 (6): 681-703.

[15] McIvor, Ronan; McCracken, Martin and McHugh, Marie (2011) "Creating outsourced shared services arrangements: Lessons from the public sector", European Management Journal, 29: 448-461.

[16] de Cuevas, Rachel; Lawson, Lovett; Al-Sonboli, Najla; Al-Aghbari, Nasher; Arbide, Isabel; Sherchand, Jeevan; Nnamdi, Emenyonu; Aseffa, Abraham; Yassin, Mohammed; Abdurrahman, Saddiq; Obasanya, Joshua; Olanrewaju, Oladimeji; Datiko, Daniel; Theobald, Sally; Ramsay, Andrew; Squire, Bertel; and Cuevas, Luis (2016) "Patients direct costs to undergo TB diagnosis", Infectious Diseases of Poverty; 5: 24-32.

[17] John, Peter (2003) "Is There Life After Policy Streams, Advocacy Coalitions, and Punctuations: Using Evolutionary Theory to Explain Policy Change?" The Policy Studies Journal, 31 (4): 481-498.

[18] Ridde, Valery (2009) "Policy Implementation in an African State: An Extension of Kingdon's Multiple-Streams Approach", Public Administration; 87 (4): 938-954.

[19] Anderson, Stuart (2012) "Public, private, neither, both? Publicness theory and the analysis of healthcare organisations", Social Science \& Medicine; 74: 313-322.

[20] Wilson D and Game C, 2011, Local government in the United Kingdom, 5thedn, Basingstoke: Palgrave Macmillan.

[21] Boehme, Catharina; Nicol, Mark; Nabeta, Pamela; Michael, Joy; Gotuzzo, Eduardo; Tahirli, Rasim; Gler, Tarcela; Blakemore, Robert; Worodria, William; Gray, Christen; Huang, Laurence; Caceres, Tatiana; Mehdiyev, Rafail; Raymond, Lawrence; Whitelaw, Andrew; Sagadevan, Kalaiselvan; Alexander, Heather; Albert, Heidi; Cobelens, Frank; Cox, Helen; Alland, David and Perkins, Mark (2011) "Feasibility, diagnostic accuracy, and effectiveness of decentralised use of the Xpert MTB/RIF test for diagnosis of tuberculosis and multidrug resistance: a multicentre implementation study" The Lancet, 377 (9776): 1495-1505. 
[22] Sachdeva, K. (2013) "Experience with implementation of Xpert MTB/RIF in India", Government of India, 16 April, accessed

http://www.stoptb.org/wg/gli/assets/html/GLI5/Experience\%2 0with\%20implementation\%20of\%20Xpert\%20MTBRIF\%20i n\%20India_16April12013.pdf, 28 December 2017.

[23] Nickols, Fred (2016) "Four Strategies for Managing Change" Distance Consulting, accessed at http://www.nickols.us/four_strategies.pdf, 30 December 2017.

[24] van Kampen, Sanne; van Cleeff, Maarten; van Gorkom, Jeroen and Rehr, Manuela (2013) "TB CARE I Core project: Intensified implementation of GeneXpert MTB/RIF in 3 Countries", TB CARE, July, accessed at http://www.tbcare1.org/publications/toolbox/tools/lab/TB CA RE_I_GeneXpert_Core_Project_Final_Report.pdf, 29 December 2017.

[25] Rehr, Manuela (2013) "Roll-out of Xpert MTB/RIF" STOP TB: 5th GLI Meeting, 16 April, accessed at http://www.stoptb.org/wg/gli/assets/html/GLI5/Rehr Xpert T B\%20CARE\%20I_final_no\%20animations_w\%20annex.pdf, 29 December $201 \overline{7}$.

[26] Dalberg (2017) "UNITAID end-of-project evaluation: TB GeneXpert - Scaling up access to contemporary diagnostics for TB" UNITAID, January-March, accessed at https://unitaid.eu/assets/TB-Xpert-Evaluation_Report-_Final.p df, 29 December 2017.

[27] WHO (2013) Monitoring of Xpert MTB/RIF roll-out in Nigeria: Country/partner reported procurement and plans, STOP TB, 2 July, accessed at http://www.stoptb.org/wg/gli/assets/documents/map/2/Pdf_file s/NGA.pdf, 29 December, 2017.

[28] Walker, Damian (2001) "Economic analysis of tuberculosis diagnostic tests in disease control: how can it be modelled and what additional information is needed?", INT J TUBERC LUNG DIS, 5 (12): 1099-1108.

[29] Andre, E.; Isaacs, C.; Affolabi, D.; Alagna, R.; Brockmann, D.; de Jong, B.; Cambau, E.; Churchyard, G.; Cohen, T.; Delmee, M.; Delvenne, J; Farhat, M.; Habib, A.; Holme, P.; Keshavjee, S.; Khan, A.; Lightfoot, P.; Moore, D.; Moreno, Y.; Mundade, Y.; Pai, M.; Patel, S.; Nyaruhirira, A.; Rocha, L.; Takle, J.; Tr'ebucq, A.; Creswell, J. and Boehme, C. (2016) "Connectivity of diagnostic technologies: improving surveillance and accelerating tuberculosis elimination", Int J Tuberc Lung Dis, 20 (8): 999-1003.

[30] Onyebuchi, Ogbonna (2015) "Tuberculosis and Leprosy Control Policy in Nigeria, 1985-2015", Elixir Pharmacy, 88: 36313-36315.

\section{Biography}

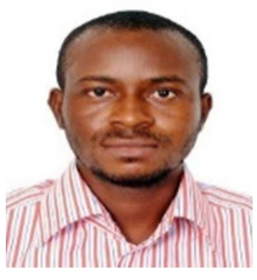

Amos Oluwasayo Akinremi trained as a medical doctor, and obtained a Master's degree in Public Health (MPH). He further acquired a Master of Public Administration (MPA) degree with Distinction from Queen Mary University of London under Commonwealth Shared Scholarship. He is a Fellow of Institute of Management Consultants (FIMC), and a Fellow of Institute for Health Insurance and Managed Care of Nigeria (FIHIMN). He is presently the Director of Planning, Research and Statistics at Ogun State Health Insurance Agency in Nigeria, and he is involved in several projects and programmes that address the critical gaps in healthcare system. 